\title{
Common mechanism controlling phase and antigenic variation in pathogenic neisseriae
}

\author{
A. Stern and T. F. Meyer* \\ Max-Planck-Institut für Biologie, Infektgenetik, \\ Spemannstrasse 34, D-74 Tübingen, FRG.
}

\section{Summary}

The expression of the Neisseria gonorrhoeae opacity protein (Op, protein II), a major antigenic determinant of the outer membrane, is subject to frequent phase transitions. At least nine expression loci (opaE) are involved in the production of a large number of serologically distinct Op types. Using opa-specific oligonucleotides as probes in genomic blots, we detect Op-related gene sequences (opr) in N. meningitidis as well as in $N$. lactamica. DNA sequence analysis of such opr genes derived from $\mathbf{N}$. meningitidis reveals distinct regions of homology with gonococcal opaE genes. As shown in the immunoblot, the proteins encoded by opa and opr are serologically related. Like the opaE genes, the 5'-coding sequences of the opr genes include a repetitive sequence composed of pentameric CTCTT units. The number of these coding repeat (CR) units is variable. This finding, together with the observation that all opr genes are constitutively transcribed, regardless of the status of protein production, suggests a translational control mechanism identical to that of the opa genes in gonococci. The related structures and control mechanisms of opa and opr genes imply a general significance of their gene products for the pathogenic character of the investigated Neisseria species.

\section{Introduction}

The Neisseria species form a group of Gram-negative diplococci that colonize the mucosal surfaces of mammals. The genus Neisseria includes both pathogenic and harmless commensal species. The pathogenic nature is strongly pronounced in $N$. gonorrhoeae and $N$. meningitidis (Bovre, 1984), but other members, too, may show opportunistic properties causing infections in a variety of anatomical sites (Johnson, 1983). The determinants that are responsible for the infectious character of certain Neisseria species are not very well understood.

Received 20 February, 1987. *For correspondence.
Bacterial virulence is a consequence of multiple attributes affecting different stages during the course of an infection. Many efforts have been made to identify specific factors that would help to distinguish between pathogenic species and commensal organisms. Indeed, some predominant characteristics have been implicated in pathogenicity, i.e., the production of an $\lg A 1$ protease (Plaut et al., 1975; Pohiner et al., 1987), the ability of bacteria to adhere specifically to mucus-secreting columnar epithelial cells (McGee et al., 1982), the presence of the so-called H. 8 antigen in the outer membrane (Cannon et al., 1984; Black and Cannon, 1985), and the expression of iron-regulated proteins (Mietzner et al., 1986).

Another series of investigations focused on variable components on the surface of pathogenic Neisseria spp., such as pili, outer membrane proteins and lipopolysaccharides, which are thought to contribute to the virulence of the organisms. Surface variability is considered as a crucial aspect of pathogenic Neisseriae which enables them to colonize different microenvironments within the host during the course of a natural infection, and to evade the host's immune response (Lambden et al., 1979; Watt and Ward, 1981; Kristiansen et al., 1984).

In Neisseria gonorrhoeae, the pilus protein as well as the opacity protein (Op, also named P.II) show significant inter- and intra-strain variability (Black et al., 1984; Blake and Gotschlich, 1983; Heckels, 1981; Swanson, 1982). In Neisseria meningitidis, intra-strain heterogeneity has been demonstrated for outer membrane proteins of class 5 and LPS (Stephens and McGee, 1983; Poolman et al., 1985). Regarding the heat-modifiable mobility during electrophoresis, the sensitivity to proteolytic enzymes, the occurrence of phase variation, and the extensive variability in expression and molecular weight, the class 5 proteins seem to be the equivalents of the Op molecules of the gonococcal outer membrane (Poolman et al., 1985).

From our studies of the opa gene system, which is responsible for Op expression in N. gonorrhoeae, we have learned that the genome of $N$. gonorrhoeae harbours at least nine gene loci, all of which are able to express variant Op molecules (Stern et al., 1984). All members of this gene family are constitutively transcribed. However, translation to a functional protein can only occur if the translational start codon ATG is in frame. The reading frame is controlled by a structural element, termed the coding repeat (CR), which consists of a variable number of CTCTT repeat units. Such units can be spontaneously added or 
lost, manoeuvring the ATG codon into or out of frame (Stern et al., 1986). In addition to its role in gene conversion, CR variation appears to be the basic mechanism involved in phase and antigenic variation of the gonococcal Op expression.

The class 5 proteins in the outer membrane of $N$. meningitidis exhibit similarities to the gonococcal Ops with regard to their heat-modifiable electrophoretic mobility and their extensive antigenic diversity (Poolman et al., 1985). This fact prompted us to extend our molecular analysis of surface variation to other interesting members of the genus Neisseria. Our report describes the structure and regulation of Op-related genes (opr) that we detected in $N$. meningitidis and also $N$. lactamica, demonstrating the narrow relatedness of the three systems.

\section{Results}

Identification of opr sequences in N. meningitidis and N. lactamica

Using conserved oligonucleotide probes of a gonococcal opa gene in a dot blot assay we recently became aware of Op-related (opr) sequences in $N$. lactamica and $N$. meningitidis, though not in other Neisseria species (unpublished results). One of the probes. AN3, was used to characterize further the opr sequences detected in the two Neisseria species by Southern blot analysis. The AN3 probe was homologous to the so-called coding repeat (CR) sequence consisting of CTCTT pentamer units. Chromosomal DNA samples prepared from one $N$. lactamica strain and four $N$. meningitidis strains, and from N. gonorrhoeae strain MS11 as a reference, were digested with $\mathrm{Clal}$ and Pst endonucleases (Fig. $1 \mathrm{~A}$ and $\mathrm{B}$, respectively) and, after blotting onto nitrocellulose, probed with labelled AN3. The results of the hybridization clearly demonstrate the presence of more than one opr gene in genomic DNA of both $N$. lactamica and $N$. meningitidis. In contrast to the complexity of the opa gene family in gonococci, only four opr loci were found in each of the $N$. meningitidis strains and no more than two were found in N. lactamica.

Op-related proteins in N. lactamica and N. meningitidis

The detection of opr loci in N. lactamica and N. meningitidis raised some questions: are these genes active in the two non-gonococcal species and, furthermore, do the gene products show significant homology to the gonococcal Op protein. In order to investigate the expression of Op-related proteins, immunoblotting was performed using rabbit antiserum raised against denatured Op protein of the gonococcal strain MS11 Vo (Stern et af., 1984). Four out five $N$. meningitidis isolates produced one protein

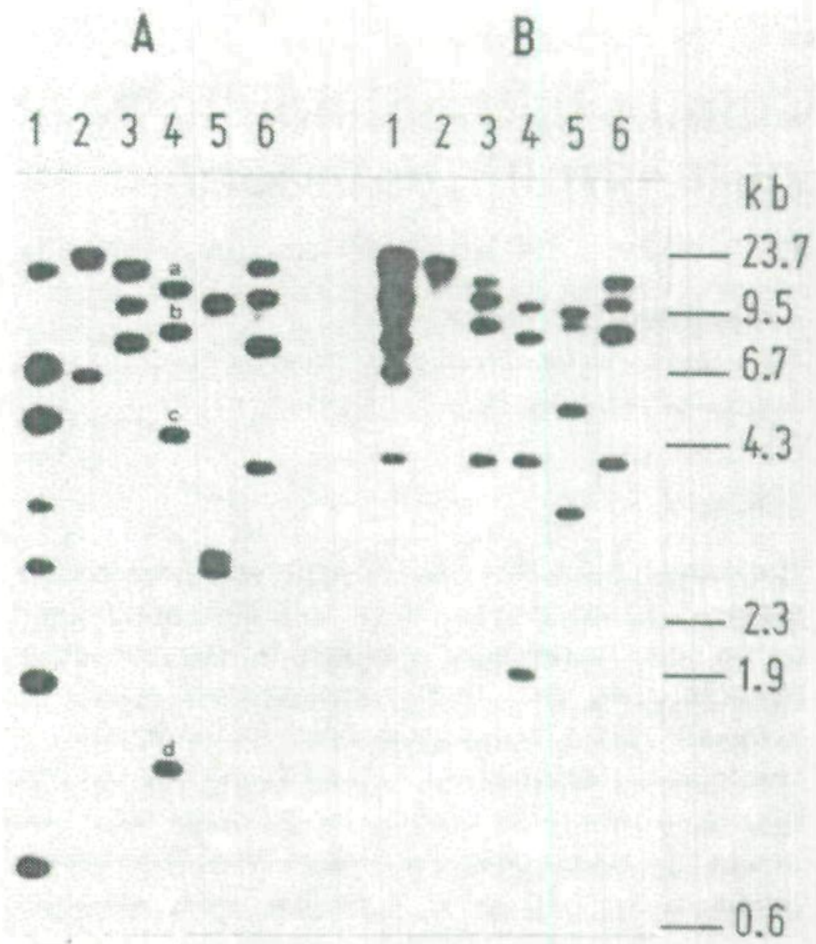

Fig. 1. Genomic biot of chromosomal DNA from different Neisseria species. Both panels $(A$ and $B$ ) show six lanes with chromosomal DNA from $N$.

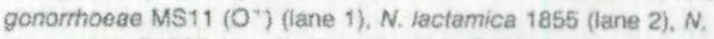
meningatidis $\mathrm{B} 1937$ (lane 3), N. meningitidis C1938 (lane 4), N. meningitidis B1940 (lane 5), and N. meningitidis AMP140 (lane 6). Genomic DNA was digested with Clal (A) and Psti (B), respectively, and probed with the oligomer AN3 (Fig. 4).

each that showed strong cross-reactivity with the anti-Op serum (Fig. 2). The molecular weights of the detected proteins corresponded to those of the class 5 proteins of $N$. meningitidis. In the cell lysates of $N$. meningitidis DMP141 and the $N$. lactamica isolate no Op-related proteins were detectable. Obviously, the number of proteins expressed did not correlate with the number of opr loci detected in the Southern blot and therefore not every locus was active in the production of a protein.

Fig. 2. Immunoblotting of Op. related proteins in cell lysates of various Neisseria species.

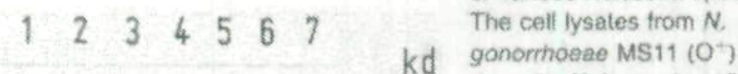
(lane 1), N. lactamica 1855

-43 (lane 2). N. meningritidis B1937 (lane 3), N. meningitidis C1938 (lane 4). N. meningitidis B1940 flane 5). N. meningitidis AMP140

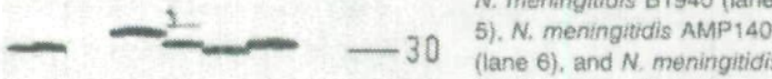
DMP141 (tane 7 ) were subjected to immunobiotting using rabbit antiserum raised against dentured Op of MS11 gonococci. 


\section{The control region of opr genes}

The identification of opr genes in N. meningitidis and $N$. lactamica using the AN3 probe at the same time demonstrated that these loci possessed a so-called coding repeat (CR) which was known to be the crucial element in the control of gonococcal opa gene expression (Stern et al., 1986). We therefore characterized the CR region of each opr locus, first by chromosomal DNA sequencing of opr genes and second, for comparison, by sequencing on the mRNA level. Both experiments were performed on the basis of the primer extension protocol successfully used previously (Stern et al., 1986). The protocol involved an oligonucleotide primer (MB1) that was complementary to a conserved sequence in opalopr genes overlapping the CR sequence and the downstream opa leader coding sequence (see Fig. 4). This primer was extended in the presence of dATP and dGTP as the sole triphosphates. Therefore, only the CTCTT pentamer repeat units of the CR sequence would be a sufficient template for DNA polymerase. The primer extension reaction was expected to be immediately terminated beyond the 5 '-end of the CR sequence, thus creating discrete bands in a sequencing gel. As a control, to exclude incidental termination, all primer extension assays were repeated with the same triphosphates plus ddTTP. In these latter assays the extended primer should have been exactly one nucleotide longer since an A was expected upstream of all CR sequences (Stern et al., 1986).

For the DNA level, Fig. 3A shows different patterns of extended primers for the various Neisseria DNAs investigated. The number of bands, each corresponding to at least one opr locus, varies between different species. Moreover, individual opr genes contain different numbers of repeat units ranging from 7 up to about 22 CF units. Some of the bands in the sequencing gel appear to be double bands, thus representing more than one opr locus. Essentially the same pattern of bands is seen on the mANA level (Fig. 3B), suggesting that all existing opr genes linked with a CR element are constitutively transcribed in the two Neisseria species.

\section{Variations in the CR region of opr genes}

In N. gonorhoeae the variability of Op-expression is controlled by the CR region which affects the translation of opa genes by switching the reading frame. To determine if the expression of individual opr genes in $N$. meningitidis and $N$. lactamica is subject to the same intriguing control mechanism, we tried to derive spontaneous isogenic varlants of some of the isolates by in vitro cultivation. This could not be done on the basis of colony morphology
A

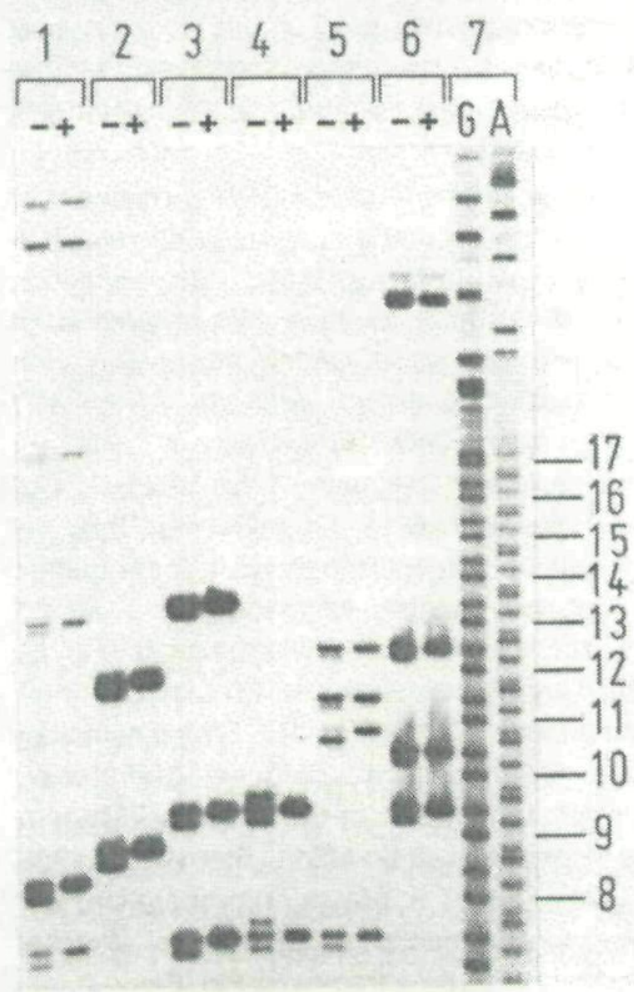

B
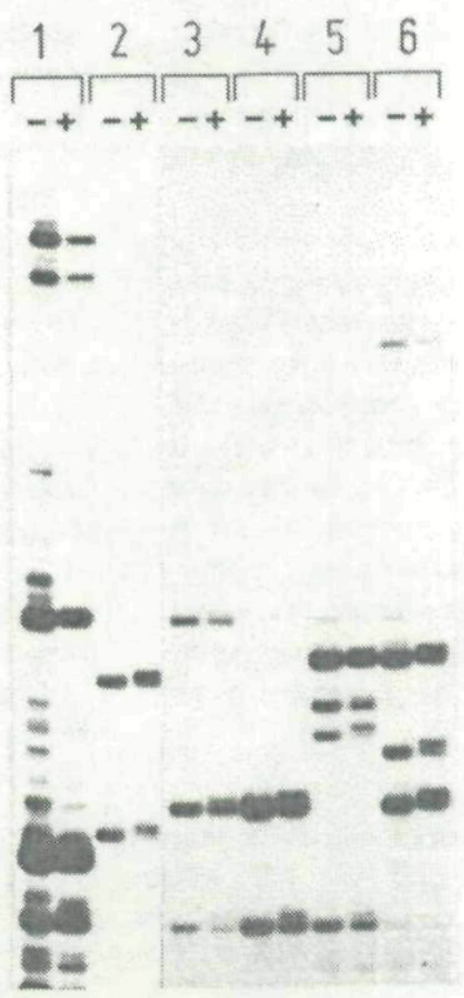

Fig. 3. Primer extension sequencing of chromosomal DNA and total RNA isolated from various Neisseria species. Chromosomal DNA (panel A) and RNA (panel B) were isolated trom $N$. gonorrhoeae MS11 $\left(\mathrm{O}^{*}\right.$ ) (lane 1). N. lactamica 1855 (lane 2), N. meningitidis B1937 (lane 3), N. meningitidis C1938 (lane 4), N. meningitidis B1940 (lane 5), and N. meningitidis AMP140 (ane 6) For sequencing, the 5 -labelled oligonucieotide MB1 was used as a primer. The reactions were pertomed in the presence of dATP and dGTP $(-)$. or with additional ddTTP $(+)$. As reference (iane 7. panei A), to show the repetitive features of the CA sequence, the $G$ and $A$ reaction of the standard sequencing protocol was performed on plasmid pOPf18 (Stern et al., 1986). 
A

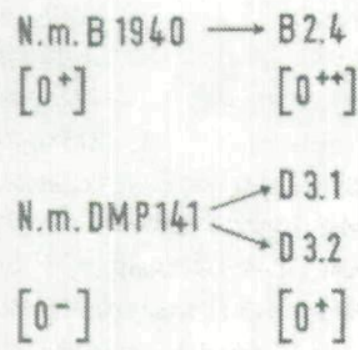

B

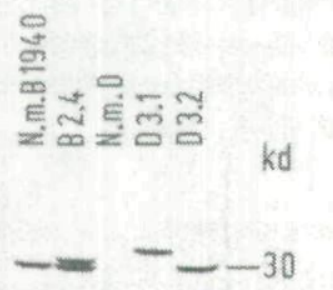

C
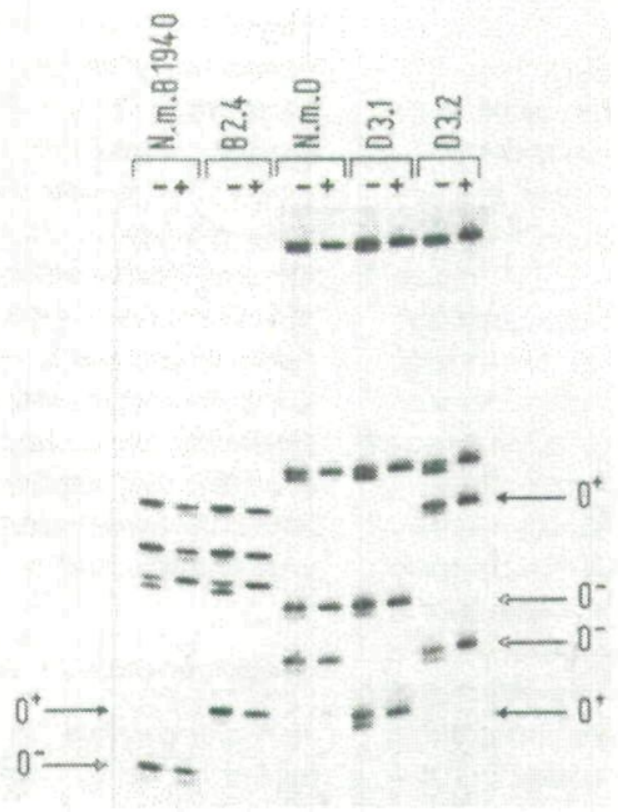

Fig. 4. Analysis of the control mechanism for phase and antigenic variation. (A) Scheme of the derivation of isoggenic variants of $N$. meningitidis strains $\mathrm{B} 1940\left(\mathrm{O}^{\circ}\right)$ and $\mathrm{DMP} 141\left(\mathrm{O}^{\circ}\right)$, (B) immunobiotting of the variant Op-related proteins in cell lysates according to the protocol in Fig. 2. (C) Prime! extension with opr-specific mRNA isolated from iscogenic meningococci. (-) and (+) reactions of the primer extension experiment are outlined in Fig. 3. The positions of the variabie bands, inciuding the putative opr expression phenotype of the corresponding transcripts, are indicated at the borders of the ge:

because the variation in Op-related proteins was not reflected by detectable differences (Pooiman et al., 1985; unpublished results).

Instead, we screened individual colonies with anti-Op serum and observed occasional changes in the reaction with this serum. Aliquots of variant colonies were taken and lysed for an immunoblot analysis. Figures $4 \mathrm{~A}$ and $\mathrm{B}$ show the derivations and the protein patterns of three spontaneous variants selected from two $N$. meningitidis strains. These are variant $B 2.4\left(\mathrm{O}^{++}: 29.5 \mathrm{kDa}, 30.5 \mathrm{kDa}\right)$ derived from the isolate $\mathrm{B} 1940\left(\mathrm{O}^{*}: 29.5 \mathrm{kDa}\right)$, and varlants $\mathrm{D} 3.1\left(\mathrm{O}^{*}: 32.5 \mathrm{kDa}\right)$ and $\mathrm{D} 3.2\left(\mathrm{O}^{+}: 30 \mathrm{kDa}\right)$ derived from isolate DMP141 $\left(\mathrm{O}^{-}\right)$.

Figure $4 \mathrm{C}$ shows the primer extension experiments done with chromosomal DNA of these isogenic meningococcal opr variants. The $\mathrm{O}^{+}$to $\mathrm{O}^{++}$switch of parental $\mathrm{B} 1940$ to variant B2.4 involves a distinct increase in size of one band, corresponding to an insertion of precisely one CR unit. Further, the two independent switches from $\mathrm{O}^{-}$to $\mathrm{O}^{*}$ of isolate DMP141 to its isogenic derivatives D3.1 and D3.2 are associated with the loss of a single CR unit in one transcript and an insertion of two CR units in another transcript. This result shows a strict correlation of opr gene expression and the variations of the $C R$ unit number, which directly affect the reading frames in the $5^{\prime}$ regions of opr genes.

\section{Cloning and DNA sequence analysis of meningococcal opr genes}

To analyse the relatedness of opa and opr structural genes we determined the DNA sequences of two opr gene copies of $N$. meningitidis C1938. For this purpose we extracted the four opr-specific Clal fragments of this strain (Fig. 1A, lane 4) out of a preparative agarose gel. As a reference, a small aliquot of this gel was subjected to Southern analysis using the AN3 probe. Before cloning, we determined the number of CR units of each individual Clal fragment by primer extension. In agreement with the primer extension experiment performed with total genomic DNA, we found for three fragments $(a, b$, and $d)$ nine CR units each and for fragment $c$ seven repeat units (Fig. 1). The two smaller fragments were inserted into the vector pBA and cloned in $E$. coli.

The physical map of the clones pOPM1 (fragment c) and POPM3 (fragment d), and the strategy of their DNA sequence analysis using synthetic oligonucleotide primers, are shown in Fig. 5; the nucleotide sequences of the two cloned meningococcal opr loci are presented in Fig. 6. Plasmid pOPM1 contains a complete opr gene of $780 \mathrm{bp}$, whereas plasmid pOPM3 harbours a partial opr gene that lacks its $3^{\prime}$-end because of an intragenic Clal site used for the digestion of the chromosomal DNA.

The DNA sequence alignment reveals a large degree of homology between the two opr genes, including the transcriptional and translational initiation signals (Fig. 6). The major sequence diversities appear to be concentrated on a few hypervariable regions (Fig. 5B). Strong homology also exists with the gonococcal opa genes (Stern et al., 1986). A stringent comparison of the amino acid level of six different opa genes of strain MS11 (Stern et al, 1986; A. Stern, F. Jahnig and T.F. Meyer, in preparation) with the two meningococcal genes reveals several stretches of continuous homology (see frames in Fig. 6). 


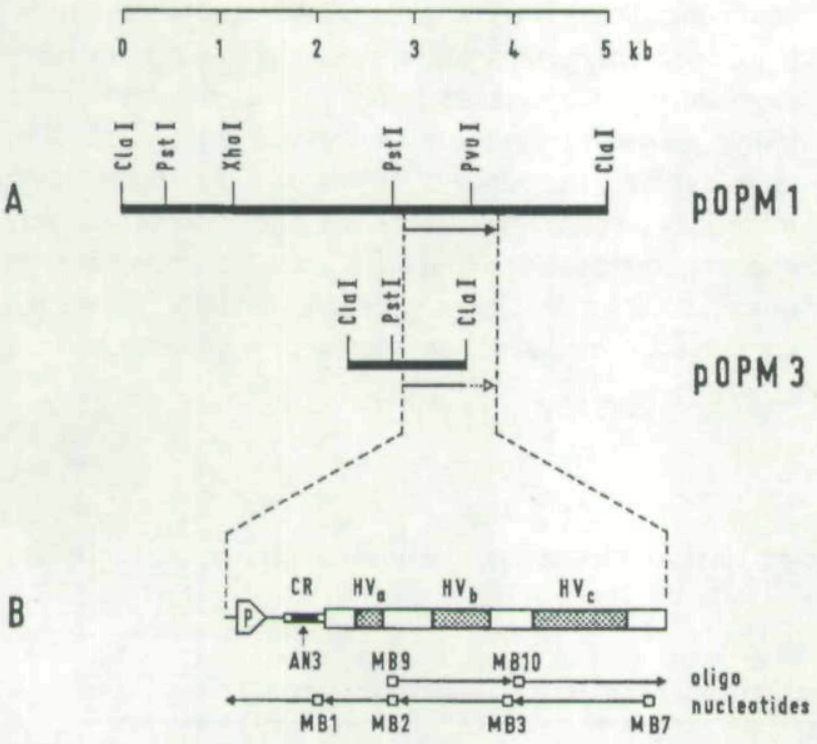

Fig. 5. Physical map and DNA sequencing strategy of the cioned $o p r_{c}$ and $o p r_{0}$ loci of $N$. meningitidis $C 1938$. (A) The top shows the physical maps of pOPM1 $\left(o p r_{c}\right)$ and pOPM3 $\left(o p r_{d}\right)$. The coding regions of the opr genes and the direction of transcription, as deduced from the sequencing data, are marked by an arrow. (B) represents a generalized diagram of the opr genes with a CR sequence, hypervariable regions (HV), and the promoter $(P)$. The location of the oligonucleotide sequences used as primers in the DNA sequencing experiments are indicated. The boxes indicate the location of the primers, the arrows designate the direction of the primer extension. AN3 was used as a probe in DNA filter hybridizations. Sequence of the oligonucleotide primers:

AN3: 5'-CTCTTCTCTTCTCTTCTCTTCTCTT-3'

MB1: 5'-TGCGCTGCGGAAGAGAAG-3'

MB2: 5'-ATGCGTACGGATGTTTCT-3'

MB3: 5'-ATAGGGTTTGAATTTATC-3'

MB7: 5 -TTCGTGGGTIITGAAGCG-3

MB9: 5'-AACATCCGTACGCATTCC-3

MB10: 5'-GTCGCCTACGGACACGTC-3'

The reading frame of both cloned opr genes is out of frame with respect to the unique initiation codon ATG (Fig. 6). This is consistent with the CR variation control model for an inactive opr expression locus (Fig. 7). However, the $o p r_{d}$ locus cloned in plasmid pOPM3 shows only $8 \mathrm{CR}$ units in contrast to the $9 \mathrm{CR}$ units expected from the genomic sequencing experiment of fragment d (Fig. 3A). Clone pOPM1, on the other hand, contains the expected number of $7 \mathrm{CR}$ units. Interestingly, when we extrapolate the pOPM3 clone to the expected 9 CR units, the gene comes into frame (Fig. 6). Because of our cloning strategy we can exclude the possibility of having cloned an opr locus other than $o p r_{d}$. Instead we must account for the loss of one CR unit to a spontaneous CR switch in opr $r_{d}$. Whether it occurred in a small fraction of the meningococcal population used for the cloning, or after cloning in E. coli, is not known. More importantly, we can assume now that $o p r_{d}$ with its original 9 units represents the active opr locus of strain C1938, being responsible for the production of the Op-related protein (Fia 2 lane 4).

\section{Discussion}

The suggested significance of gonococcal Op in virulence and the intriguing genetic system involved in the expression of this protein led us to extend our interest on Oprelated proteins to other Neisseria species. By Southern blotting we revealed two opr gene loci in $\mathrm{N}$. lactamica and four loci in each of the meningococcal isolates investigated. Although this study shows a much lower complexity of opr genes in these two species as compared with the gonococcal opa gene family which has at least nine gene loci, nevertheless, the typical regulatory features of gonococcal opa genes appear to be conserved in the opr genes of $N$. lactamica and $N$. meningitidis: i.e. (a) all opa and opr genes present in the genome are constitutively transcribed, (b) the 5'-regions of these genes are composed of pentameric repeat units, the so-called CR units that code for the hydrophobic part of a leader peptide, (c) the number of CR units is cifferent in most of the genes of one genome and, finally, (d) the variation of opa and $o p r$ expression is inherently associated with changes in the CR unit number of individual transcripts.

Fig. 7 illustrates the control mechanism of opa/oprgenes that appears to be a common principle in the three different Neisseria species investigated. In all three species at least two expression loci are found that can be activated to produce Op and Op-related proteins. The number of CR units present in an individual gene locus determines whether translation into a functional protein can occur. As the majority of the opalopr gene sequences code for variable proteins, changes in the repeat number of different gene loci can result in both phase and antigenic variation.

It is conceivable that the number of opalopr genes present in a single genome directly influences the frequency of phase transitions. Nonetheless, the opr system in N. lactamica, harbouring only two gene loci, may be efficient enough to deceive the immune system of the host, provided that these genes can be expressed. A comparable less complex variation system could be found in Salmonella typhimurium where the production of only two antigenic types of the flagella is controlled by an invertable element (Simon et al., 1980).

The presence of opr sequences in N. lactamica, commonly regarded as a commensal, is consistent with a number of reports that $N$. lactamica can occasionally induce infections at different sites in humans (Johnson, 1983). Furthermore, thermal renaturation studies have shown that $N$. gonorrhoeae, N. meningitidis and N. lactamica share greater DNA homology with each other than with other Neisseria species (Hoke and Vedros, 1982). In addition, the expression of $\mathrm{H} .8$ antigen and the AgR-37K protein in the three species supports the assumption that an evolutionary divergent development separated them from the other members of the genus (Cannon et al., 1984; Black and Cannon, 1985; Mietzner et al., 1986). 
The opa and opr genes share conserved coding sequences. Previous investigations indicate that the meningococcal class 5 proteins, also subject to an extensive variability, represent the equivalent of the opacity proteins in N. gonorrhoeae (Frasch and Chapman, 1972; Frasch and Mocca, 1978; Ashton et al., 1983; Poolman et al., 1985). Convincing evidence for the relatedness of the two protein classes was demonstrated by their aminoterminal sequence homology (M. Achtmann, personal communication). The complete DNA sequence of a meningococcal opr gene, presented here, shows that the homology of the two classes is not restricted to the amino terminus. Apart from the hypervariable regions, the Oprelated protein sequence of $N$. meningitidis, representing a class 5 protein, shows only few additional amino acid exchanges in positions that are conserved among several opacity proteins of $N$. gonorrhoeae strain MS11. The strict conservation of distinct structures of a variable surface

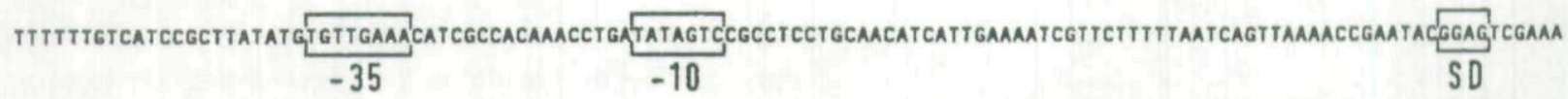

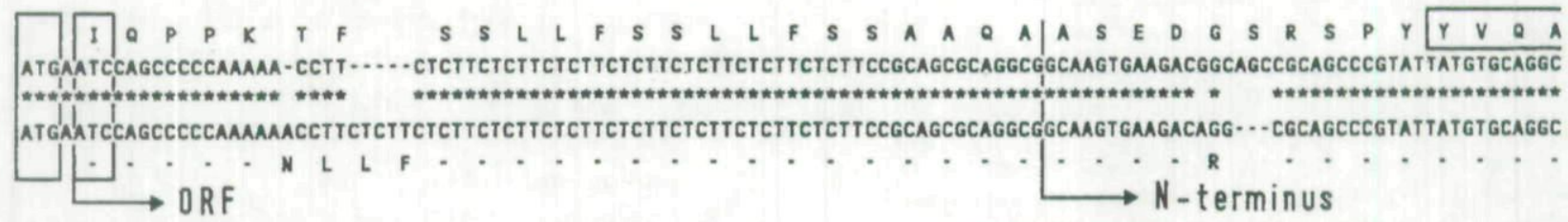

D L A Y Y A A E E R I T H W GGATTTAGCCTATGCCGCCGAACGCATIACCCACAATTATCCGgAACCAACCGgTGCAGACAAAGACAMAATAMGCACAGTAAGCGATTATTTCAGAAACATCCGTGCGCATTCCATCCA *******************************************************

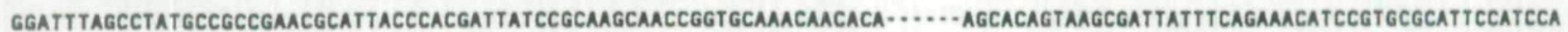

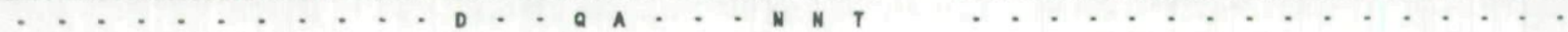

P R R V S S V V G G Y Y D D F F G G G W CCCCAGGGTGTCGTCGGCTACGATTTCGGCGGCTGGAGGATAGCGGCAGATTATGCCAGTTACAGAAAATGgAAAGAAAGTAATTTTCTACTAAAAAAGTTACTGAAGAGATAAAAGA

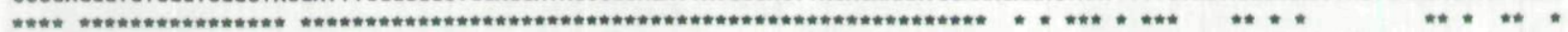

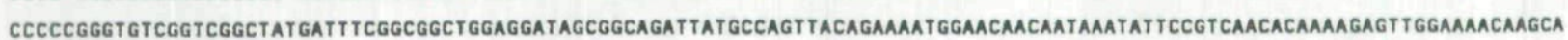
-

N Y K E T K T E H Q G N G S F H A T S S S L G L S CAACTACAAAGAAACCAAAACAGAACATCAAGgAaACGGCAGC -...TTCCACGCCACTTCTTCTCTCGGCTTATCCGCCATTTACGATTTCAAACTCAACGATAAAT TCAAACCCTATAT

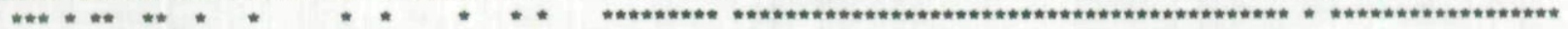
TAACAATAAGAAAGACCTGAAGACGGAAAATCAGgAAAACGGTACATTCCACGCCGCTTCTTCTCTCGGCTTATCCGCCATTTACGATTTCAAACTCAAGGGTAAATTCAAACCCTATAT

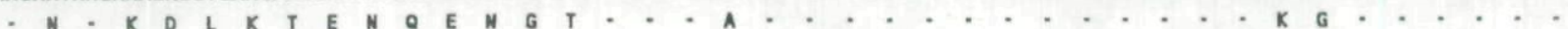

G A $R$ R $V$ V CGGTGCGCGCGTCGCCTACGgGCACGTTAAACATCAGGTTCATTCGGTGGAAACAAAAACCACGACTGTTACCTCTAAACCAAAGGGGGCACTCCAGCTGGAGGCCCTGTTATAAAAAC

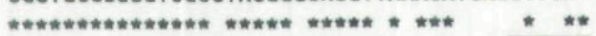
cggtgcgcgcgtcgcttacggacacgtcagacacagcatcgat] [la I

-...........

D P S $S$ P P $Y$ H TGATCCCAGCAAACCTCCCTATCACGAAAGCCACAGCATCAGCAGCTTGgGTCTTGGTGTCATCGCCGGTGTCGGTTTCGACATCACGCCCAAGCTGACTTTAGACACCGGATACCGCTA

H N W W G G CCACAACTGGGGACGCTTGGAAAACACCCGCTTCAAAACCCACGAAGCCTCATTGGGCATGCGCTACCGCTTCTGA

\section{ORF}

Fig. 6. DNA sequences of the opr genes cloned in DOPM1 and pOMB3. The upper DNA and protein sequences refer to pOPM1, the lower ones to pOPM3. Asterisks between the two sequences indicate homology on the DNA level. Upstream of the open reading frame (ORF) and the ATG initiation codon (vertical frames), typical -35 and -10 promoter regions and a Shine-Daigarno (SD) sequence are marked by horizontal frames. With respect to the ORF the ATG initiation codon is out-of-frame in both gene loci. Homology with conserved opa regions of $N$. gonorrhoeae strain MS11 is indicated by horizontal frames at the protein level. 


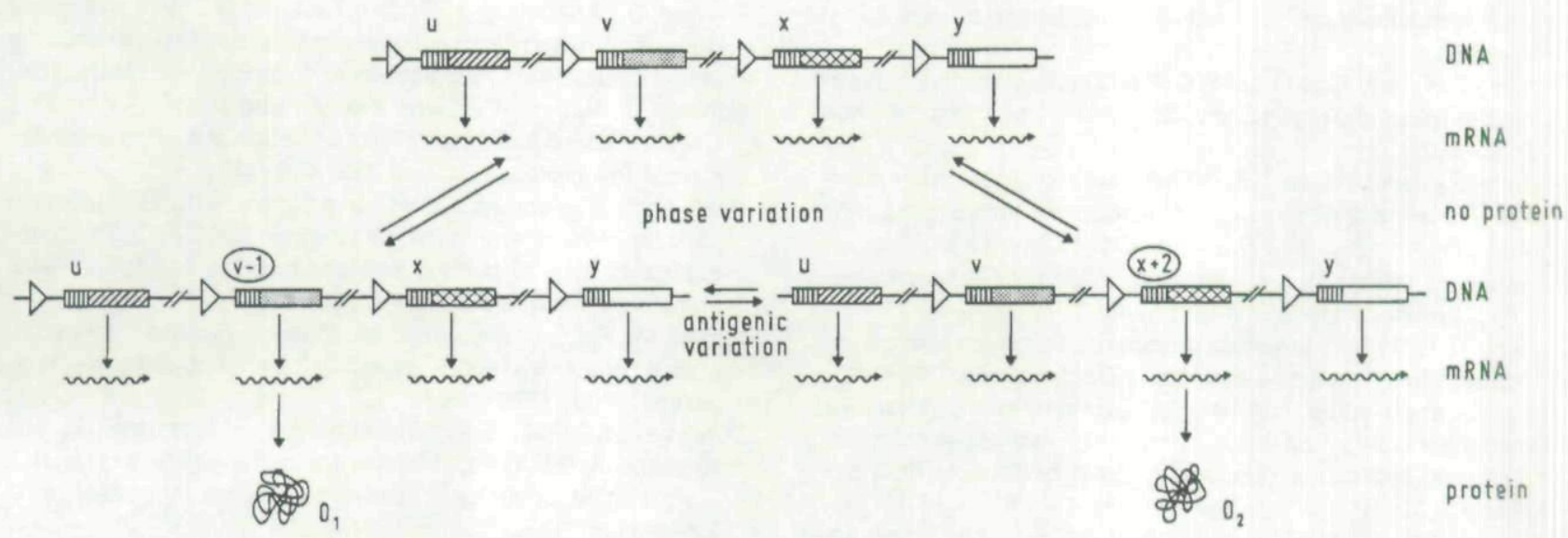

Fig. 7. Model for the control of phase and antigenic variation (for explanations see Discussion).

protein among pathogenic Neisseria species might provide an essential function associated with the pathogenic character of these bacteria.

\section{Experimental procedures}

\section{Bacterial strains}

N. meningitidis AMP140, B1937, B1940, C1938, DMP141 of the respective serotypes $A$ to $D$, and $N$. lactamica 1855 were kindly provided by U. Berger. N. gonorrhoeae strain MS11 (Stern et al., 1986) was obtained from E. C. Gotschlich. The opa and opr expression phenotypes are indicated by $O *$. E. coli strains GC1 (Meyer et al., 1982) and DH1 (Hanahan, 1983) were used for cloning of meningococcal DNA.

\section{Primer extension and sequencing}

$5 \mu \mathrm{g}$ of chromosomal- or $100 \mathrm{ng}$ of gel-purified DNA were digested with $M$ bol restriction enzyme. The DNA was ethanol-precipitated, and dissolved in $3 \mu \mathrm{l} \mathrm{H} \mathrm{H}_{2}$ and $4 \mu \mathrm{l}$ of $10 \mathrm{mM} \mathrm{NaOH}$. The DNA was denatured by heating to $100^{\circ} \mathrm{C}$ and the solution was neutralized with $2 \mu / 20 \mathrm{mM}$ Tris- $\mathrm{HCl} \mathrm{pH} 7.6$ and $1.5 \mu / 10 \mathrm{mM} \mathrm{HCl}$. 1.5 pmoles of $5^{\prime}-\mathrm{P}^{32}$-labelled MB1 primer were coupled to the DNA by incubating at $42^{\circ} \mathrm{C}$ for 5 min after a second heating. The annealed sample was supplemented with $10 \mathrm{mM}$ Tris- $\mathrm{Hcl} \mathrm{pH} 8.3$, $5 \mathrm{mM} \mathrm{MgCl}_{2}, 200 \mathrm{mM}$ dGTP and $200 \mathrm{mM}$ dATP and split into two aliquots. The second aliquot was supplemented with additional $400 \mathrm{mM}$ ddTTP. $1 \mathrm{U}$ Klenow polymerase was used to extend the primer for $30 \mathrm{~min}$ at room temperature. For primer extension with total mRNA cloned $200 \mathrm{U}$ M-MLV reverse transcriptase (BRL) and 0.5 pmol end-labelled oligomer MB1 were used per assay (Stern et al., 1986). The meningococcal clones pOPM1 and $\mathrm{POPM} 3$ were sequenced by a modified supercoil method using end-labelled oligonucleotides as primers (Wallace et al., 1981).

\section{Other techniques}

For detection of Op-related proteins in non-gonococcal strains, $100 \mu \mathrm{g}$ of total cell lysate solved in sample solution was electrophoresed on a $12.5 \%$ polyacrylamide gel and blotted onto nitrocellulose sheets. To extract DNA from gels, the agarose pieces were put into dialysis tubings and electro-eluted in $40 \mathrm{mM}$ Tris-acetate $\mathrm{pH} 8$ and $1 \mathrm{mM}$ EDTA. The immunoblotting assay (Blake et al., 1984), the immunological screening of colonies (Stern et al., 1984), DNA filter hybridization (Stern et al., 1984) and oligonucleotide preparations (Stern et al., 1986) have been described.

\section{Acknowledgements}

We are grateful for the excellent assistance in these experiments by $S$. Störzbach, for the preparation of oligonucleotides by $B$. Tries and J. Pohlner, and for the artwork by K. Lamberty. This work was supported by the Deutsche Forschungsgemeinschatt (grant Me705/2-3).

\section{References}

Ashton, F.E., Ryan, J.A., Jones, C., Brodeur, B.R., and Diena, B.B. (1983). Serotypes of Neisseria meningitidis associated with an increased incidence of meningitidis cases in Hamilton area, Ontario, during 1978 and 1979. Can J Microbiol 29: 129-136.

Black, W.J., Schwalbe, R.S., Nachamkin, I., and Cannon, J.G. (1984) Characterization of Neisseria gonorrhoeae protein II phase variation by use of monoclonal antibodies. Infect Immun 45(2): 453-457.

Black, W.J., and Cannon, J.G. (1985) Cloning of the gene for the common pathogenic neisseria $\mathrm{H} .8$ antigen from Neisseria gonorrhoeae. Infect Immun 47(1): 322-325.

Blake, M.S., and Gotschlich, E.C. (1983) Gonococcal membrane proteins: speculation on their role in pathogenesis. Prog 33: 298-313.

Blake, M.S., Johnston, K.H., Russell-Jones, G.J., and Gotschlich, E.C. (1984) A rapid, sensitive method for detection of alkaline phosphatase-conjugated anti-antibody on western blots. Anal Biochem 136: 175-179.

Bovre, K. (1984) Family VIII. Neisseriaceae. In Bergey's Manual of Systematic Bacteriology. Kreig, N.R. and Holt, J.G. (eds). Baltimore, The Williams \& Wilkins Co., pp. 288-310.

Cannon, J.G., Black, W.J., Nachamkin, I., and Stewart, W. (1984). Monoclonal antibody that recognizes an outer membrane antigen common to the pathogenic neisseria species but not to 
most nonpathogenic neisseria species. Infect Immun 43(3): 994-999.

Frasch, C.E., and Chapman, S.S. (1972) Classification of Neisseria meningitidis group B into distinct serotypes. Infect Immun 5: 98-102.

Frasch, C.E., and Mocca, L.F. (1978) Heat-modifiable outer membrane proteins of Neisseria meningitidis and their organization within the membrane. J Bacteriol 136: 1127-1134.

Hanahan, D. (1983) Studies on transformation of Escherichia coli with plasmids. J Mol Biol 166: 557-580.

Heckels, J.E. (1981) Structural comparison of Neisseria gonorrhoeae outer membrane proteins. J Bacteriol 145: 736-742.

Hoke, C., and Vedros, N.A. (1982) Taxonomy of the Neisseria: deoxyribonucleic acid base composition, intraspecific transformation, and desoxyribonucleic acid hybridization. Int J Sys Bacteriol 32: 57-66.

Johnson, A.P. (1983) The pathogenic potential of commensal species of Neisseria. J Clin Pathol 36: 213-223.

Kristiansen, B.E., Sorensen, B., Simonsen, T., Spanne, O., Lund, V., and Bjorvatn, B. (1984) Isolates of Neisseria meningitidis from different sites in the same patient: phenotype and genomic studies, with special reference to adherence, piliation, and DNA restriction endonuclease pattern. J Infect Dis 150: 389-396.

Lambden, P.R., Heckels, J.E., James, L.T., and Watt, P. (1979) Variations in surface protein composition associated with virulence properties in opacity types of Neisseria gonorrhoeae. $J$ Gen Microbiology 114: 305-312.

McGee, Z.A., Stephens, D.S., Melly, M.A., Gregg, C.R., Schlech, W.F., and Hoffman, L.H. (1982) in Microbiology 1982. Schlessinger, D. (ed.). Washington D.C.: American Society for Microbiology.

Meyer, T.F.. Mlawer, N., and So, M. (1982) Pilus expression in Neisseria gonorrhoeae involves chromosomal rearrangement. Cell 30: 45-52.

Mietzner, T.A., Barnes, R.C., Jeanlouis, Y.A., Shafer, W.M., and Morse, S.A. (1986) Distribution of an antigenically related ironregulated protein among the Neisseria spp. Infect Immun 51: $60-68$.
Plaut, A.G., Artenstein, M.S., and Capra, J.D. (1975) Neisseria gonorrhoeae and Neisseria meningitidis: extracellular enzyme cleaves human immunoglobulin A. Science 190: 1103-1105.

Pohiner, J., Halter, R., Beyreuther, K., and Meyer, T.F. (1987) Gene structure and extracellular secretion of Neisseria gonorrhoeae IgA protease. Nature 325: 458-462.

Poolman, J.T., Hopman, C.T.P., and Zanen, H.C. (1985) Colony variants of Neisseria meningitidis strain 2996 (B:26:P1.2): influence of class 5 outer membrane proteins and lipopolysaccharides. J Med Microbiol 19: 203-209.

Simon, M., Zieg. J., Silverman, M., Mandell, G., and Doolittle, R. (1980) Phase variation: evolution of a controlling element. Science 209: 1370-1374.

Sorensen, B., Falk, E.S., Wislotf-Nilsen, E., Bjorvatn, B., and Kristiansen, B.E. (1985) Multivariate analysis of Neisseria DNA restriction endonuclease patterns. J Gen Microbiol 131: 3099-3104.

Stephens, D.S., and McGee, Z.A. (1983) Association of virulence of Neisseria meningitidis with transparent colony type lowmolecular-weight outer membrane proteins. J Infect Dis 147: 282-292.

Stern, A., Nickel, P., Meyer, T.F., and So, M. (1984) Opacity determinants of Neisseria gonorrhoeae: gene expression and chromosomal linkage to the gonococcal pilus gene. Cell 37: 447-456.

Stern, A., Brown, M., Nickel, P., and Meyer, T.F. (1986) Opacity genes in Neisseria gonorrhoeae: control of phase and antigenic variation. Cell 47: 61-71.

Swanson, J. (1982) Colony opacity and protein II compositions of gonococci. Infect Immun 37: 359-368.

Wallace, R.B., Johnson, M.J., Suggs, S.V., Miyoshi, K., Bhatt, R., and Itakura, K. (1981) A set of synthetic oligodeoxyribonucleotide primers for DNA sequencing in the plasmid vector pBR322. Gene 16: 21-26.

Watt, P.J., and Ward, M.E. (1981) In Bacterial Adherence. Beachey, E. (ed.). New York: Chapman and Hall, pp. 255-288. 
This document is a scanned copy of a printed document. No warranty is given about the accuracy of the copy. Users should refer to the original published version of the material. 